\title{
Rizartrosis y síndrome del canal carpiano: tratamiento simultáneo a través de una misma vía de abordaje
}

\author{
P. Sánchez Gómez ${ }^{(1)}$ A. Fuentes Díaz ${ }^{(2)}$, F. Lajara Marco ${ }^{(3)}$, \\ S. Arlandis Villarroya ${ }^{(1)}$, F. J. Ricón $\operatorname{Recarey}^{(1)}$ \\ Servicio COyT. Hospital Vega Baja de ORIhUela (ALICANTE) \\ (1) FEA DE COYT \\ (2) JEFE DE SECCIÓN Y SERVICIO COYT \\ (3) MIR DE COYT
}

Correspondencia:

Dr. Plácido Sánchez Gómez

Ctra. Orihuela-Amoradí, s/n

03314 San Bartolomé (Orihuela). Alicante

Teléfono 965877500

e-mail: placidosanchezgomez@yahoo.es

\begin{abstract}
Objetivo: La asociación de rizartrosis y síndrome del canal carpiano es frecuente. El objetivo de este estudio es valorar el resultado de la descompresión del canal carpiano a través de la vía utilizada para realizar la artroplastia de resección-suspensión del trapecio, así como discutir las ventajas e inconvenientes encontrados con esta técnica.

Material y método: Entre 2002 y 2006 se realizaron 16 artroplastias de resección-suspensión que presentaban un síndrome del canal carpiano asociado. Se valoró la resolución de la sintomatología mediante escala analógica visual, la recuperación de la fuerza de agarre de puño y las pinzas pulgar-índice, y la mejoría producida por la intervención en la calidad de vida de los pacientes utilizando la versión española de los cuestionarios DASH (Disabilities of the Arm, Shoulder, and Hand) y CTS (Carpal Tunnel Syndrome).
\end{abstract}

Resultados: Se revisaron 14 pacientes con un seguimiento medio de 23,7 meses (rango: 10-52).

La sintomatología mejoró 8 puntos de media tras la intervención. La fuerza final de agarre de puño y de las pinzas pulpejo-lateral y pulpejo-pulpejo fueron $54,5 \%, 71,8 \%$ y $73,5 \%$ de los valores de referencia según edad, género y lado afecto.

El cuestionario DASH mejoró 34,8 puntos, y el CTS 0,8 puntos para la valoración funcional y de 2,5 puntos para la gravedad de síntomas.
Introduction: Trapeziometacarpal joint osteoarthritis is common associated with carpal tunnel syndrome.

The aim of the present study was to investigate the concomitant treatment of carpal tunnel syndrome and trapeziometacarpal joint osteoarthritis employing the same surgical incision.

Material and methods: From 2002 to 2006 we performed 16 combined thumb carpometacarpal arthroplasty and radial approach carpal tunnel release through a single incision.

Preoperative and postoperative evaluation of subjective and objective sings and symptoms was also obtained through visual analog scale, grip and pinch strengths, and CTS and DASH score.

Results: At mean follow-up of 23,7 months, we observed decreased pain of 8 points in VAS. Final grip and lateral and tip pinch strength were 54,5\%, $71,8 \%$, and $73,5 \%$ to the normal rates.

DASH score decreased 34,8 points and CTS 0.8 and 2,5 points.

Discussion and conclusion: Carpal tunnel syndrome release may be performed before, during or after surgery for trapeziometacarpal joint osteoarthritis. Because unrecognised carpal tunnel syndrome can result in additional postoperative pain and weakness and even precipitate reflex sympathetic dystrophy following thumb carpometacarpal ar- 
Discusión y conclusiones: Aunque el tratamiento del canal carpiano puede realizarse antes, durante o después de la cirugía de la artrosis trapecio-metacarpiana, para disminuir la morbilidad postoperatoria y la persistencia de síntomas se recomienda que ambas patologías sean tratadas en el mismo acto quirúrgico, y si fuera posible a través de la misma incisión. No alarga significativamente la intervención, permite una descompresión efectiva con mínima morbilidad y evita algunas de las complicaciones asociadas a la técnica tradicional.

Palabras clave: rizartrosis, síndrome del canal carpiano, tratamiento quirúrgico. throplasty, we advocate that carpal tunnel release should be performed at the same time. To avoid the added morbidity of a second palmar incision, we have begun using a radial approach, utilising the same incision we use for the thumb arthroplasty. This approach is simple, safety and effectiveness.

Key words: trapeziometacarpal joint osteoarthritis, carpal tunnel syndrome, treatment.

\section{INTRODUCCIÓN}

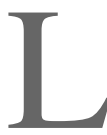

a rizartrosis presenta una fuerte asociación a otras patologías como artrosis escafo-trapecio-trapezoidea, síndrome del canal carpiano (SCC), deformidad en hiperextensión del pulgar, artrosis metacarpofalángica, pulgar en resorte, enfermedad de De Quervain y tenosinovitis ${ }^{1-3}$. Esto hizo que Melone et al. ${ }^{4}$ en 1987 las incluyesen en el denominado síndrome de la articulación basal del pulgar («basal joint syndrome»), explicando su origen por la existencia de un proceso inflamatorio de los tejidos circundantes.

El SCC es una patología frecuente, con una incidencia estimada en la población general entre el $0,1 \%$ y el $9,2 \%$, según las series consultadas $^{5}$. Es más frecuente en el grupo demográfico de mujeres por encima de los 50 años y coexiste con la rizartrosis en el $18-46 \%$ de pacientes $^{6}$. Las causas de esta frecuente asociación no están bien establecidas y se postulan distintas teorías: una que la artrosis TMC podría alterar la anatomía ósea del canal carpiano aumentando la presión en su interior, otra la disminución del diámetro antero-posterior del canal descrita en pacientes con rizartrosis por un colapso mediocarpiano, una tercera la atribuye a la inflamación TMC propagada a la vaina tenosinovial del canal carpiano, y por últi- mo hay quien la explica por una atrofia secundaria a una compresión del nervio mediano que podría sobrecargar la articulación $\mathrm{TMC}^{6}$.

Clásicamente, y aún hoy, la mayoría de cirujanos, tratan estas dos patologías mediante incisiones separadas ${ }^{4-7}$. Una incisión radial para la trapeciectomía y posterior reconstrucción ligamentosa con interposición tendinosa, y otra volar y ligeramente cubital para la descompresión del nervio mediano.

Aunque el SCC puede ser tratado antes, durante o después de la cirugía de la TMC, deberían ser tratados en el mismo acto quirúrgico y si fuera posible a través de la misma incisión quirúrgica para reducir el tiempo de cirugía, disminuir la morbilidad postoperatoria, evitar la persistencia de síntomas y mejorar la apariencia estética ${ }^{8-10}$.

El propósito de este trabajo es analizar los resultados de la descompresión del canal carpiano a través de la vía utilizada para realizar la artroplastia de resección-suspensión del trapecio, así como discutir las ventajas e inconvenientes encontrados con esta técnica.

Los objetivos principales de esta revisión fueron evaluar el efecto de la cirugía sobre el alivio del dolor, la amplitud de movimiento, la fuerza, la mejoría de la función física, la evaluación subjetiva por parte del paciente y las complicaciones. 

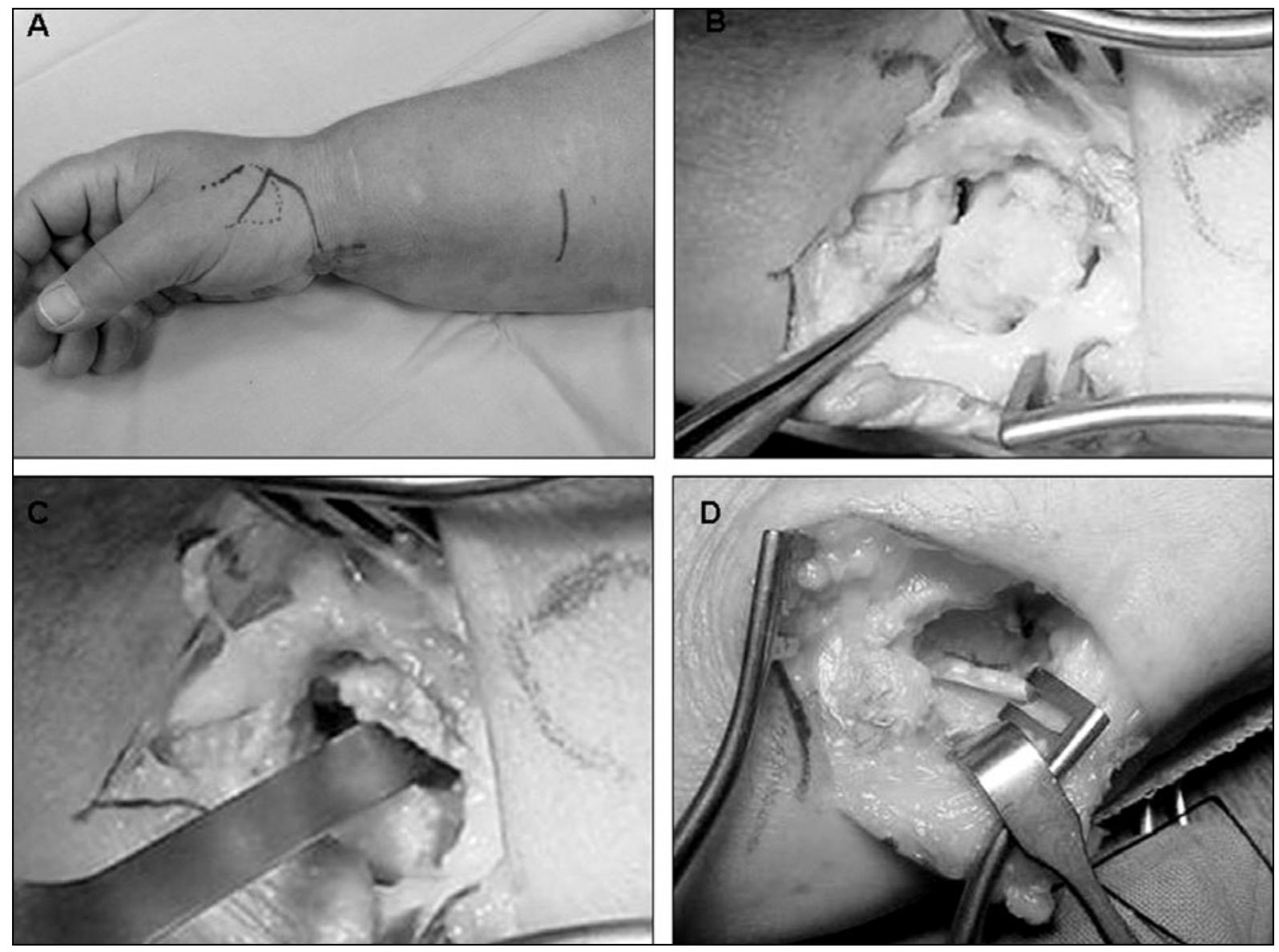

Figura 1. Técnica quirúrgica (I). Se realiza una incisión en zig-zag sobre la zona dorso-radial de la base del pulgar (A). Se incide la capsula articular entre el tendón del abductor pollicis longus (APL) y el del extensor pollicis brevis $(\mathrm{EPB})(\mathrm{B})$. Se realiza la exéresis del trapecio con cuidado de no dañar el tendón del FCR (C). Con la exéresis del trapecio se produce la apertura de la capa superficial del retináculo flexor quedando a la vista el tendón FCR (D).

\section{MATERIAL Y MÉTODO}

Entre mayo de 2002 y abril de 2006 se realizaron 45 artroplastias de resección-suspensión, 19 de ellas presentaban un SCC asociado en el momento del diagnóstico.

Como criterios para pertenecer al estudio, se incluyeron todos los pacientes con diagnóstico de rizartrosis ${ }^{1-3}$ (dolor TMC, grinding test $+\mathrm{y}$ radiología compatible) que no respondieron a tratamiento conservador con antiinflamatorios, férulas e inyección de corticoides y presentaban simultáneamente una compresión del nervio mediano a nivel del canal carpiano. El diagnóstico de SCC se estableció apoyándose en los síntomas y $\operatorname{signos}^{5}$ (dolor nocturno o por el día, hormigueos y parestesias, debilidad o atrofia de la musculatura tenar, disminución de la discri- minación de dos puntos, Tinel y Phalen positivos), y en la electromioneurografía (EMG). La anormalidad en la EMG, la historia clínica compatible y al menos un síntoma mayor o unas pruebas de provocación positivas, establecieron el diagnóstico 5 .

Se utilizaron como criterios de exclusión, la sospecha de SCC postraumático, la pérdida sensitiva o atrofia muscular grave y los antecedentes de cirugía previa de SCC.

Se incluyeron 16 de los 19 pacientes (Tabla I), 15 mujeres $(93,8 \%)$ y 1 hombre $(6,2 \%)$ de edades comprendidas entre 48 y 72 años (edad media 59,8 años), con afectación del lado derecho en 7 ocasiones $(43,8 \%)$ siendo la mano no dominante la intervenida en el $62,5 \%$ de las ocasiones (10 pacientes). A todos se les pasó una escala analógica visual del dolor y cuestionarios 


\section{Tabla I - MATERIAL Y MÉTODO}

\begin{tabular}{|c|c|c|c|c|c|}
\hline Casos & Género & Edad & $\begin{array}{l}\text { Lado afecto } \\
\text { (Dominante) }\end{array}$ & $\begin{array}{l}\text { Estadío } \\
\text { artrosis }^{*}\end{array}$ & Profesión \\
\hline 1 & $\mathrm{H}$ & 72 & (D) & IV A & Agricultor \\
\hline 2 & M & 67 & I & III A & Ama de casa \\
\hline 3 & M & 62 & (D) & III A & Agricultora \\
\hline 4 & $M$ & 76 & I & III A & Ama de casa \\
\hline 5 & M & 48 & I & $\| \quad L$ & Limpiadora \\
\hline 6 & M & 57 & I & III A & Ama de casa \\
\hline 7 & $M$ & 56 & I & III C & Cocinera \\
\hline 8 & M & 52 & (D) & III A & Ama de casa \\
\hline 9 & M & 60 & (D) & IV & Hostelería \\
\hline 10 & M & 58 & (D) & IV & Almacén \\
\hline 11 & M & 48 & I & III & Cocinera \\
\hline 12 & M & 58 & (D) & III & Ama de casa \\
\hline 13 & M & 60 & D & III L & Limpiadora \\
\hline 14 & M & 61 & I & IV & Hostelería \\
\hline 15 & M & 58 & I & III & Agricultora \\
\hline 16 & M & 65 & I & $\| A$ & Ama de casa \\
\hline
\end{tabular}

$\mathrm{H}$ : hombre

M: mujer

D: derecha

I: izquierda

* Clasificación de Eaton y Littler ${ }^{11}$

DASH y CTS prequirúrgicos como parte del protocolo de primera visita en consultas externas.

En todos los casos la artroplastia se realizó utilizando un hemitendón del flexor carpi radialis (FCR) según técnica de Burton-Pellegrini modificada y la apertura del canal carpiano a través de una vía transtrapezoidea ${ }^{12}$. Bajo anestesia regional endovenosa o plexo braquial y manguito de isquemia en raíz del miembro, se realiza una incisión en zig-zag sobre la zona dorso-radial de la base del pulgar. Se incide la capsula articular entre el tendón del abductor pollicis longus (APL) y el del extensor pollicis brevis (EPB), se realiza la exéresis del trapecio con cuidado de no dañar el tendón del FCR. Con la exéresis del trapecio se produce la apertura de la capa superficial del ligamen- to transverso del carpo quedando a la vista el tendón FCR. Se retrae volarmente dicho tendón $y$, por la ventana dejada tras la exéresis del trapecio, se secciona la capa profunda del ligamento transverso del carpo en el borde radial, ayudados de un clamp para proteger las estructuras intracanal, siguiendo la dirección oblicua de $45^{\circ}$ del FCR, con lo que se puede observar en el interior del canal carpiano el tendón del flexor pollicis longus (FPL) y la sinovial flexora. Este paso puede realizarse asistido por artroscopia, permitiendo una mejor visualización y exploración del canal. Una vez descomprimido se continúa con la artroplastia de suspensión utilizando un hemitendón del FCR. Tras realizar hemostasia y cierre por planos, se coloca vendaje algodonado y férula antebraquial con inmovilización del primer dedo, y se retira el manguito de isquemia. La sutura se retira a los 10-15 días y la férula se mantiene 4-6 semanas.

Se registró el aumento que ocasionaba la descompresión nerviosa en la duración de la intervención, así como la incidencia de complicaciones intra y postoperatorias.

Se valoró la sintomatología dolorosa mediante una escala analógica visual (EVA) de 0 a 10 puntos, la discriminación de dos puntos (distancia mínima que debe haber entre dos alfileres para que el sujeto experimente dos sensaciones táctiles distintas), la amplitud de movimiento del pulgar (abducción radial o apertura de la comisura en grados y nivel de oposición a $5^{\circ}$ dedo), la fuerza de agarre de puño y las pinzas pulgar-índice mediante dinamómetros de JAMAR y B\&L respectivamente ${ }^{13-15}$. Además se calculó la mejoría producida por la intervención en la calidad de vida de los pacientes utilizando la versión española de los cuestionarios DASH (Disabilities of the Arm, Shoulder, and Hand) ${ }^{16-17}$ y CTS (Carpal Tunnel Syndrome) ${ }^{16,17}$ determinando su respuesta en función al «tamaño del efecto» (ES) (media de la diferencia prepostratamiento/ desviación estándar de la línea base) y en relación también a la Media Estandarizada de la respuesta o SRM (media de la diferencia/desviación estándar de la diferencia $)^{18}$, definiendo el cambio como pequeño cuando era $\leq 0,2$, medio $\leq 0,5$ y grande $\geq 0,8^{19}$.

Siguiendo la teoría de Dubert y Kamoun ${ }^{20}$ del tamizado («tamisage»), los resultados se di- 

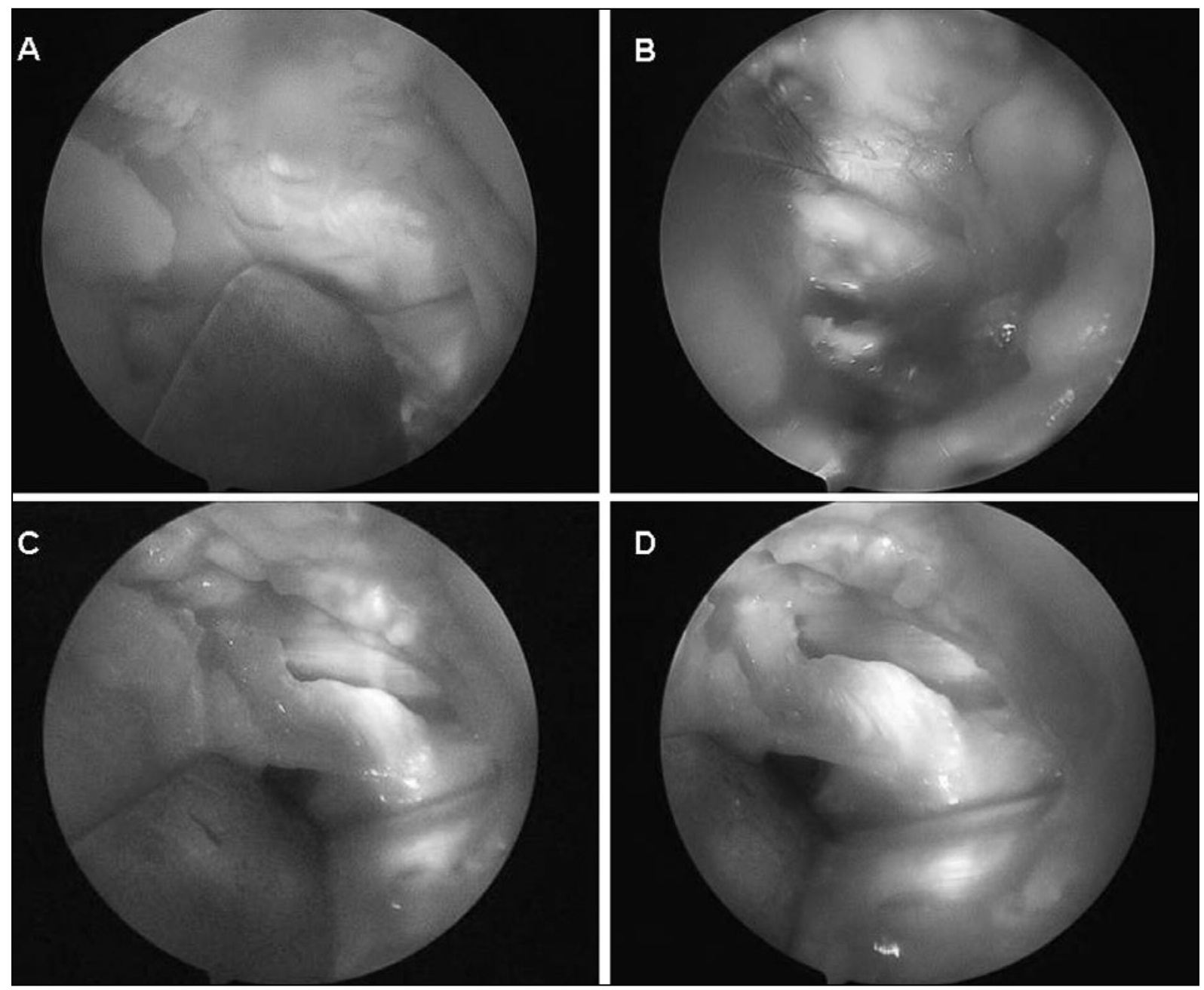

Figura 2. Técnica quirúrgica (II). Se retira volarmente dicho tendón (A), y se secciona la capa profunda del ligamento transverso del carpo en el borde radial siguiendo la dirección oblicua de $45^{\circ}$ del FCR (B), con lo que se puede observar en el interior del canal carpiano el tendón del flexor pollicis longus (FPL) y la sinovial flexora. (C, D). Este paso puede realizarse asistido por artroscopia para una mejor visualización.

vidieron en tres grupos o categorías: la valoración subjetiva por parte de los pacientes (estableciendo como resultado malo una diferencia entre el DASH pre y postquirúrgico inferior a 10 puntos), la valoración clínica por parte del facultativo (considerando como resultado malo la persistencia de dolor o parestesias, o la existencia de complicaciones tendinosas o nerviosas), y la valoración socioprofesional por parte de la sociedad (determinando un mal resultado la no reinserción laboral). Un resultado malo en cualquiera de estas categorías se consideró un fallo del tratamiento al final del período de seguimiento.

\section{RESULTADOS}

Tras un seguimiento medio de 23,7 meses (rango: 10-52) se revisaron 14 de los 16 pacientes (los dos pacientes restantes fueron considerados como perdidas de seguimiento y se excluyeron del estudio). Tablas II y III.

La duración media para la realización de la artroplastia de suspensión-interposición con hemitendón del FCR y la apertura del canal carpiano por la misma vía fue de 77 minutos (rango: 57-120), siendo el incremento medio en la duración de la intervención por la descompresión del SCC de 3.3 minutos de media (rango: 1-7). 
Tabla II - Resultados (I)

\begin{tabular}{|c|c|c|c|c|c|c|c|c|c|c|c|c|c|}
\hline & & Dolor & & & Fue & rza pu & uño & & uerza & & & Fuerza & PP \\
\hline Caso & $\begin{array}{l}\text { Seguimient } \\
\text { (meses) }\end{array}$ & $\begin{array}{c}\text { Parestesias } \\
\text { pre/post }\end{array}$ & $\begin{array}{c}\text { Abducción } \\
\left.\text { radial ( }{ }^{\circ}\right)\end{array}$ & Oposición & $\begin{array}{c}\text { afecto/no } \\
\text { afecto }\end{array}$ & $\begin{array}{c}\% \\
\text { teórico }\end{array}$ & $\begin{array}{c}\% \\
\text { contralateral }\end{array}$ & $\begin{array}{c}\text { afecto/no } \\
\text { afecto }\end{array}$ & $0 \%$ & $\begin{array}{c}\% \\
\text { contralateral }\end{array}$ & $\begin{array}{l}\text { afecto/no } \\
\text { afecto }\end{array}$ & $\begin{array}{c}\% \\
\text { teórico }\end{array}$ & $\begin{array}{c}\% \\
\text { contralateral }\end{array}$ \\
\hline 1 & 20,1 & $6 / 0$ & 50 & IFP & $26 / 21$ & 66,6 & 8, 123 & $8 / 6$ & 100 & 133,3 & $8 / 6$ & 126,9 & 133,3 \\
\hline 2 & 27 & $10 / 2,2$ & 50 & IFP & $4 / 9$ & 16,6 & 44,4 & $4 / 5$ & 72,7 & 80 & $2 / 4$ & 41,6 & 50 \\
\hline 3 & 12,2 & $8 / 4$ & 40 & IFP & $25 / 19$ & 98 & 131,5 & $7 / 4,5$ & 5112,9 & 64,2 & $2 / 4$ & 43,4 & 50 \\
\hline 4 & 19 & $10 / 2,0$ & 40 & IFP & $8 / 6$ & 38,1 & 133,3 & $2 / 3$ & 39,2 & 155,5 & $1 / 4$ & 23,8 & 25 \\
\hline 5 & 44,5 & $10 / 0$ & 40 & MF & $18 / 16$ & 64,2 & 112,5 & $5 / 5$ & 76,9 & 100 & $5 / 5$ & 90,9 & 100 \\
\hline 6 & 52 & $10 / 0$ & 50 & IFP & $11 / 4$ & 42,3 & 275 & $3 / 2$ & 51,7 & 150 & $3 / 2$ & 63,8 & 150 \\
\hline 7 & 32,3 & $8 / 3,5$ & 70 & MF & $8 / 10$ & 30,7 & 80 & $5 / 6$ & 86,2 & 83,3 & $5 /$ & 106,3 & 166,6 \\
\hline 8 & 19,1 & $10 / 0$ & 40 & MF & $32 / 32$ & 123 & 100 & $6 / 7$ & 103,4 & 85,7 & $7 / 7$ & 122,8 & 100 \\
\hline 9 & 21 & $10 / 3$ & 70 & MF & $4 / 10$ & 14,2 & 40 & $2 / 3$ & 30,7 & 66,6 & $2 / 4$ & 43,4 & 50 \\
\hline 10 & 21,3 & $7 / 0$ & 40 & MF & $14 / 12$ & 50 & 116,6 & $5 / 7$ & 76,9 & 71,4 & $4 / 4$ & 75,4 & 100 \\
\hline 11 & 23,2 & $8 / 0$ & 70 & MF & $16 / 16$ & 57,1 & 100 & $5 / 5$ & 76,9 & 100 & $3 / 3$ & 54,5 & 100 \\
\hline 12 & 19,2 & $10 / 1$ & 40 & IFP & $12 / 25$ & 42,8 & 48 & $2 / 5$ & 30,7 & 40 & $2 / 6$ & 37,7 & 33,3 \\
\hline 13 & 10,7 & $10 / 0$ & 70 & MF & $20 / 20$ & 76,9 & 100 & $5 / 5$ & 86,2 & 100 & $5 /$ & 108,6 & 100 \\
\hline 14 & 10 & $10 / 0$ & 60 & MF & $10 / 4$ & 41,6 & 250 & $3 / 2$ & 54,5 & 150 & $4 / 2$ & 88,8 & 200 \\
\hline Media & 20,16 & $9,07 / 1,12$ & 52,14 & & $4,85 / 14,57$ & 54,48 & 118,23 & $4,42 / 4,67$ & $7 \quad 71,37$ & $7 \quad 98,58$ & $3,78 / 4,21$ & 173,47 & 97,02 \\
\hline DST & & & & & $8,48 / 8,24$ & & & $1,89 / 1,63$ & & & $2,04 / 1,47$ & & \\
\hline
\end{tabular}

Pre: prequirúrgico - Post: postquirúrgico - PL: pulpejo-lateral - PP: pulpejo-pulpejo - DST: desviación estándar

La evaluación del dolor postoperatorio mostró una reducción desde los 9.1 puntos de media preoperatorios a los 1.1 puntos de media finales. Se observó un aumento del número de pacientes sin dolor (ningún paciente antes de la cirugía y 8 al final del período de seguimiento) y una disminución del número de pacientes con dolor máximo ( 9 pacientes antes de la cirugía y ninguno al final del período de seguimiento).

Se produjo una recuperación en la discriminación de dos puntos a una distancia inferior a $6 \mathrm{~mm}$ en todos los casos.

La abducción radial media del pulgar fue de $52,1^{\circ}$ (rango $40-70^{\circ}$ ), presentando 8 de los 14 pacientes $(57 \%)$ una oposición completa hasta la base del $5^{\circ}$ dedo y el resto de pacientes hasta la interfalángica proximal.

La fuerza media de las pinzas pulpejo-pulpejo y pulpejo-lateral entre pulgar e índice fueron, respectivamente, un $73,5 \%$ y un $71,4 \%$ de la esperada según tablas de normalidad para edad, género, lado afecto y dominancia ${ }^{14}, 15 ; \mathrm{y}$ de un $97 \%$ y un $98,6 \%$ respecto al lado contralateral. La fuerza final de agarre de puño o prensión fue un $54,5 \%$ de la esperada según tablas de normalidad para edad, género, lado afecto y dominancia $^{14,15}$, y un $118,2 \%$ respecto al lado contralateral.

El DASH mejoró una media de 34,8 puntos (rango: 17-85), con un ES de 1,9 y una SRM de 1,7 . 


\section{Tabla III - Resultados (II)}

\begin{tabular}{|cccccccc|}
\hline Caso & $\begin{array}{c}\text { Seguimiento } \\
\text { (meses) }\end{array}$ & $\begin{array}{c}\text { DASH } \\
\text { pre/post }\end{array}$ & $\begin{array}{c}\text { CTS RF } \\
\text { pre/post }\end{array}$ & $\begin{array}{c}\text { CTS SS } \\
\text { pre/post }\end{array}$ & $\begin{array}{c}\text { Reinserción } \\
\text { laboral }\end{array}$ & $\begin{array}{c}\text { Valoración } \\
\text { intervención }\end{array}$ & $\begin{array}{c}\text { Resultado } \\
\text { final }^{*}\end{array}$ \\
\hline 1 & 20,1 & $48 / 7$ & $1,6 / 1,3$ & $3,0 / 1$ & Sí & 10 & Bueno \\
\hline 2 & 27 & $49 / 23$ & $3,9 / 1,5$ & $3,4 / 1,1$ & Sí & 10 & Bueno \\
\hline 3 & 12,2 & $82 / 64$ & $4,4 / 4,3$ & $4,8 / 2,7$ & No & 5 & Malo \\
\hline 4 & 19 & $46 / 29$ & $2,6 / 3$ & $2,9 / 1,9$ & Sí & 7 & Bueno \\
\hline 5 & 44,5 & $82 / 20$ & $3,5 / 1,6$ & $4,5 / 1,8$ & Sí & 8 & Bueno \\
\hline 6 & 52 & $59 / 55$ & $2,5 / 2,5$ & $4,6 / 1,5$ & Sí & 8 & Malo \\
\hline 7 & 32,3 & $55 / 36$ & $3,6 / 3,4$ & $4,9 / 1,8$ & Sí & 5 & Bueno \\
\hline 8 & 19,1 & $32 / 2$ & $3,1 / 2,3$ & $4,3 / 1,8$ & Sí & 10 & Bueno \\
\hline 9 & 21 & $84 / 50$ & $3,3 / 2$ & $5 / 2,1$ & No & 5 & Malo \\
\hline 10 & 21,3 & $44 / 2$ & $1,8 / 1,8$ & $4,5 / 1,1$ & Sí & 8 & Bueno \\
\hline 11 & 23,2 & $43 / 12$ & $2,6 / 1,5$ & $3,5 / 1,4$ & Sí & 10 & Bueno \\
\hline 12 & 19,2 & $60 / 16$ & $2,5 / 1,1$ & $4,5 / 1,4$ & Sí & 7 & Bueno \\
\hline 13 & 10,7 & $85 / 0$ & $2,8 / 2$ & $4,1 / 1,6$ & Sí & 10 & Bueno \\
\hline 14 & 10 & $84 / 50$ & $3,3 / 2$ & $5 / 2,1$ & Sí & 10 & Bueno \\
\hline Media & 20,2 & $60,9 / 26,1$ & $3,0 / 2,2$ & $4,2 / 1,7$ & & 7,8 & \\
\hline DST & & $18,7 / 21,6$ & $0,8 / 1,0$ & $0,8 / 0,5$ & & & \\
\hline & & & & & & & \\
\hline
\end{tabular}

RF: resultado funcional - SS: gravedad de los síntomas - Pre: prequirúrgico - Post: postquirúrgico DST: desviación estándar - * Según Dubert y Kamoun ${ }^{20}$

El cuestionario CTS para la valoración funcional descendió con la intervención desde los 3 puntos preoperatorios a los 2.2 puntos al final del período de seguimiento con un ES de 1 y una SRM de 0.9 , y con respecto a la gravedad de síntomas desde 4,2 puntos a 1,7 puntos de media con un ES de 3.3 y una SRM de 3,8 .

De los 11 pacientes en edad laboral, 9 $(81,8 \%)$ volvieron a su ocupación previa, siendo la valoración subjetiva media de la intervención, al final del período de seguimiento, de 7,8 puntos en una escala de 0 a 10 puntos.

En total se establecieron, según Dubert y Kamoun $^{20}$, un 78,5\% de buenos resultados (11 casos) y un $21.5 \%$ de resultados insuficientes o malos (3 casos): 1 paciente donde la diferencia entre el DASH pre y postquirúrgico fue inferior a 10 puntos (aunque no presentaba sintomatología y la reinserción laboral fue completa) y 2 pacientes que no volvieron a su anterior ocupación laboral.

Como complicaciones postoperatorias encontramos, un caso de disestesia transitoria a nivel de la cicatriz quirúrgica; un granuloma a cuerpo extraño por un punto de sutura y una enfermedad de De Quervain que se solucionaron quirúrgicamente; y no hubo ningún caso de complicaciones por hipersensibilidad de la cicatriz, síndrome de pillar-pain, lesiones transitorias del nervio mediano, ni se produjo ningún caso de síndrome doloroso regional complejo. 


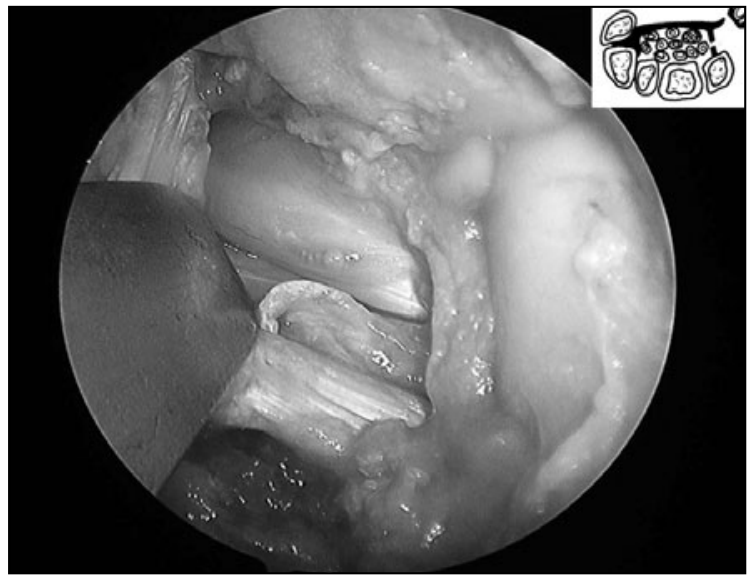

Figura 3. Técnica quirúrgica (III). Detalle ampliado y esquema de la apertura completa de la porción más profunda de la inserción radial del ligamento transverso del carpo desde el tubérculo del escafoides, con el FCR retraído volarmente en primer plano y el FPL en el interior del canal carpiano liberado.

\section{DISCUSIÓN}

La asociación de rizartrosis y síndrome del canal carpiano es frecuente en mujeres sin antecedentes traumático, con artrosis generalizada, diabéticos y pacientes que buscan una compensación económica, con una prevalencia de hasta el $43 \%{ }^{6}$. Aunque el tratamiento del canal carpiano puede realizarse antes, durante o después de la cirugía de la trapecio-metacarpiana, se recomienda que para disminuir la morbilidad postoperatoria y la persistencia de síntomas, ambas patologías sean tratadas en el mismo acto quirúrgico, y si fuera posible a través de la misma incisión quirúrgica ${ }^{8-10}$. Puesto que un SCC no reconocido resulta en un dolor postoperatorio adicional, debilidad e incluso síndrome doloroso regional complejo tras artro-
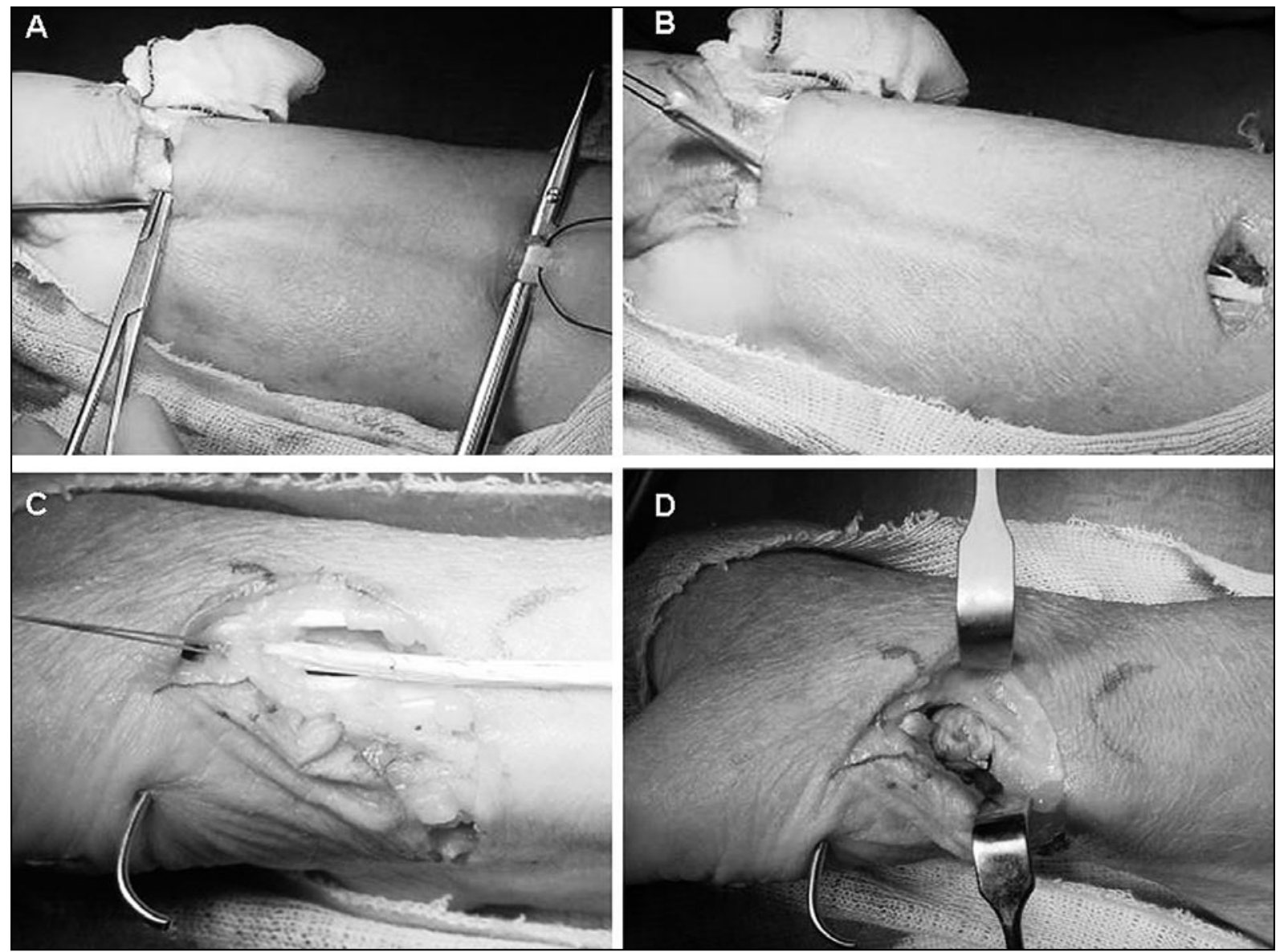

Figura 4. Técnica quirúrgica (IV). Artroplastia de suspensión-interposición tipo Burton-Pellegrini modificada. Localización del FCR a través de dos miniincisiones volares (A). Extracción del hemitendón (B). Realización de la suspensión con anclaje transóseo a base del primer metacarpiano $(C)$ e interposición del resto del hemitendón del FCR en la cavidad dejada por el trapecio (D). 


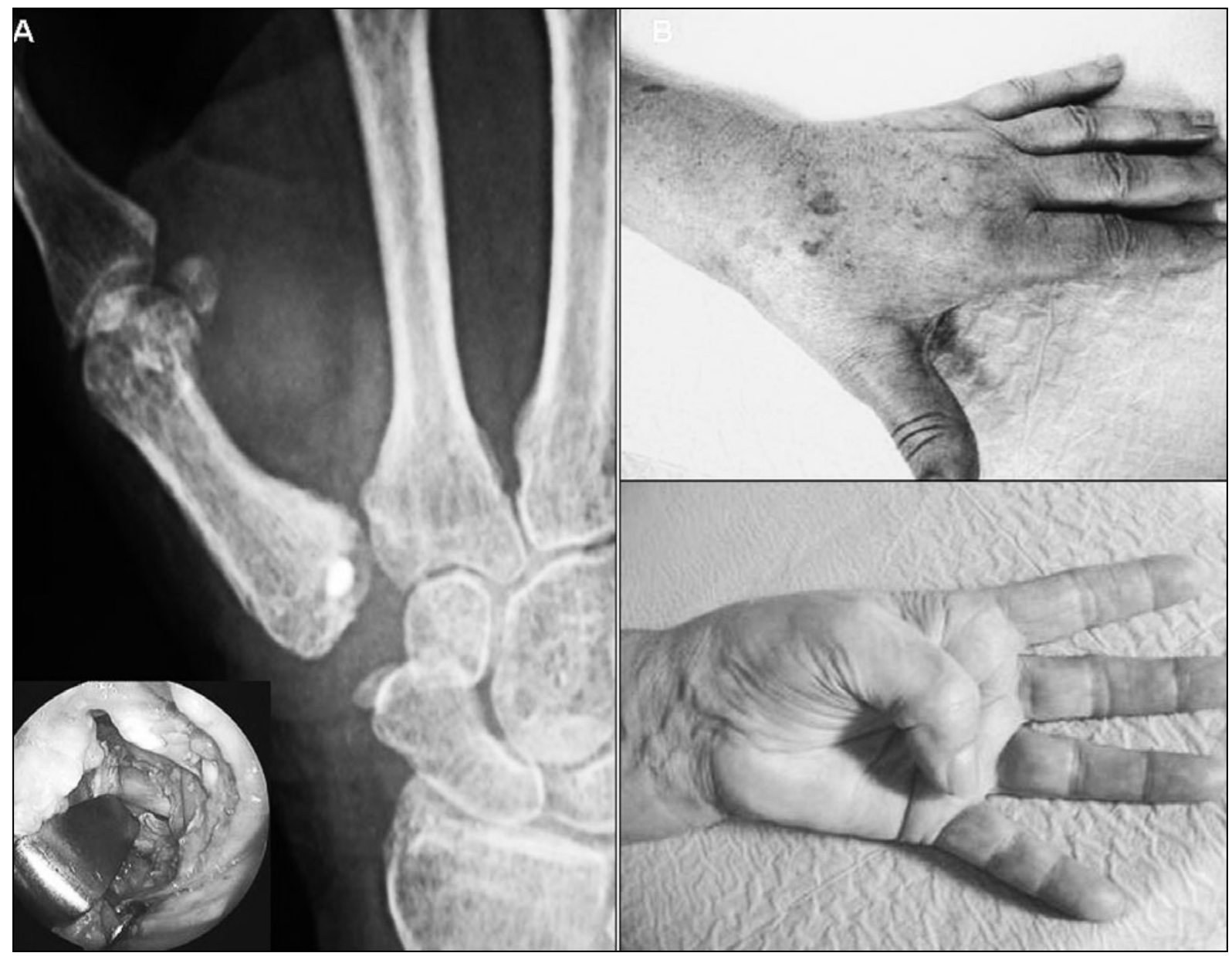

Figura 5. Caso 7: paciente de 56 años, cocinera, donde se realizó artroplastia de suspensión-interposición tipo BurtonPellegrini modificada y apertura del canal del carpo a través de la misma vía (A). Obsérvese la abducción radial de aproximadamente $70^{\circ}$ y la oposición a nivel de la metacarpofalángica del $5^{\circ}$ radio.

plastia TMC, algunos autores preconizan que la descompresión carpiana debería hacerse al mismo tiempo (Burton ${ }^{12}$ et al., Florack ${ }^{6}$ et al. y Melone et $\mathrm{al}^{4}{ }^{4}$ ). La descompresión del canal carpiano a través de una vía radial se basa en las relaciones anatómicas del retináculo flexor: sus inserciones radiales incluyen la cresta del trapecio y la tuberosidad del escafoides, dividiéndose en 2 capas separadas por el tendón del $\mathrm{FCR}^{21-23}$. La sección de éstas tras la escisión del trapecio permite la descompresión del canal carpiano, como demostraron Cassidy et al. ${ }^{9}$ en 2004, sobre 4 especímenes cadavéricos, en los que aumentaron la presión en el interior del canal carpiano hasta los $30 \mathrm{mmHg}$ con un balón percutáneo y comprobaron mediante un transductor como descendía unos $7 \mathrm{mmHg}$ de media tras la trapeciectomía y hasta la línea basal con la completa apertura del retináculo flexor a nivel del tubérculo del escafoides.

La primera referencia encontrada en la literatura sobre la utilización simultánea de una misma vía para la artroplastia de interposiciónsuspensión de la TMC del pulgar y la descompresión de un SCC concomitante la realizó A. Lluch $^{24}$ en 1994, siendo la técnica similar a la nuestra.

A partir de estos trabajos iniciales, Stahl y Shapira $^{8}$ en 2003 presentaron un estudio clínico con 20 pacientes ( 25 casos) con rizartrosis y SCC asociados tratados simultáneamente a través de la misma vía mediante esta técnica. Se trataba de 20 mujeres con una edad media de 57 años y 5 hombres con 54 años de media. El 


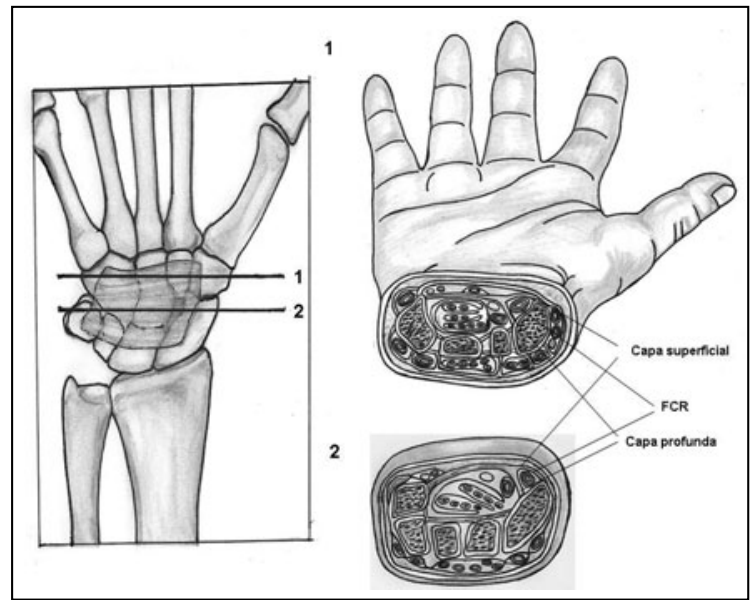

Figura 6. Relaciones anatómicas del retináculo flexor, inserciones radiales en la cresta del trapecio y la tuberosidad del escafoides, dividiéndose en 2 capas separadas por el tendón del FCR, proporcionándole un canal propio separado del canal carpiano. La sección de éstas tras la escisión del trapecio permite la descompresión del canal carpiano.

seguimiento fue de 27 meses de media y presentaron 20 resoluciones completas, 4 incompletas y 1 caso donde no se obtuvo mejoría; sin complicaciones importantes.

Finalmente, Sless y Sampson ${ }^{25}$ en 2007 presentaron los resultados de la misma técnica sobre 15 casos, con un seguimiento de 38 meses y una mejoría del dolor y parestesias completa en 14 casos e incompleta en el restante.

Nuestros resultados están en consonancia a los encontrados en la literatura, con altas tasas de buenos resultados, y escasas y poco importantes complicaciones. La mayoría de las series, incluida la nuestra, son retrospectivas, pequeñas y a veces de corto seguimiento, sin presentar grupo control, confiriendo importantes limitaciones para la extracción de conclusiones sólidas.

Entre las ventajas de esta técnica destacan que, la descompresión del nervio mediano se realiza extrafocalmente (el nervio permanece cubierto por el retináculo flexor, con lo que disminuye el riesgo de formación de cicatrices a su alrededor con las subsiguientes adherencias y dolor por tracción), la menor disrupción de la piel palmar y de partes blandas con disminución del pillar-pain, y que la realización de la trapeciectomia no se ve condicionada por la liberación del canal carpiano, sien- do el riesgo de complicaciones bajo y poco importantes ${ }^{8-10,24-25}$.

Como inconvenientes, podemos destacar que con esta vía es difícil explorar el canal carpiano porque normalmente la zona más distal y cubital del nervio mediano y su rama motora no se visualizan, por lo que no debe ser usado en pacientes con pérdida grave de la sensibilidad, marcada debilidad de la musculatura tenar, pacientes que requieran una revisión del SCC o que tengan anormalidades anatómicas ${ }^{8-10,24-25}$. Pensamos que las potenciales complicaciones por lesión de las ramas sensitivas del nervio radial, rama cutánea palmar del nervio mediano y rama superficial de la arteria radial son similares a la de la trapeciectomía aislada.

Algunos autores piensan que la morbilidad de una segunda incisión palmar para descomprimir el canal carpiano es mínima, especialmente con el uso de las técnicas endoscópicas o mini-invasivas, presentando buenos resultados a largo plazo (Florack ${ }^{6}$ et al., Melone ${ }^{4}$ et al.). Sin embargo, DaSilva el al. ${ }^{26}$, en un artículo sobre disecciones anatómicas, concluyen que las pequeñas terminaciones nerviosas de los tejidos blandos descansan profundamente sobre la zona más superficial del ligamento transverso del carpo, pudiendo dañarse cuando se utilizan tanto métodos abiertos como endoscópicos. La lesión de estas ramas terminales puede producir disestesias postquirúrgicas, hipersensibilidad de la cicatriz y pillar-pain. Martin et al. ${ }^{27}$ tras la disección de 25 manos cadavéricas, concluyeron que no existía un verdadero plano internervioso en la palma de la mano a través del cual el canal carpiano pudiera ser abordado de forma segura sin lesionar los nervios cutáneos que lo cruzan. Whealey et al. ${ }^{28}$ demostraron mediante biopsia que al menos el $50 \%$ del tejido dividido como parte de una liberación abierta del túnel carpiano era tejido nervioso.

Por último, autores como Le Viet et al. ${ }^{7}$ preconizan el uso de una vía única volar para el tratamiento de la rizartrosis y SCC siguiendo la vía del FCR descrita por Weber y Sanders ${ }^{10}$, aunque sus resultados arrojan altas tasas de complicaciones por lesión de la rama cutánea palmar del nervio mediano y síndrome doloroso regional complejo. 
En resumen, pensamos que se trata de una técnica de escasa dificultad y fácil de aprender, que no incrementa significativamente la duración de la intervención, mejora los signos y síntomas de SCC en el mismo grado que otras técnicas abiertas o endoscópicas, la tasa de complicaciones graves no es mayor que con un método estándar, evita la morbilidad de una segunda incisión en la piel y partes blandas palmares y consigue una mejor apariencia estética, descomprime al nervio mediano extrafocalmente por lo que el nervio permanece cubierto por el ligamento transverso del carpo y alejado de la cicatriz, disminuyéndose la formación de adherencias y la neuritis por tracción.

\section{BIBLIOGRAFÍA}

1. Wajon A, Ada L, Edmunds L. Surgery for thumb (trapeziometacarpal joint) osteoarthritis. Cochrane Database Syst Rev, 2005; 19: CD004631.

2. Zancolli EA, Zancolli ER, Cagnone JC. Rizartrosis del pulgar. Tratamiento quirúrgico en estadios iniciales y tardíos. Rev Iberamer Cir Mano, 2000; 27: 8-18.

3. Young SD, Mikola EA. Thumb carpometacarpal arthrosis. J Am Soc Surg Hand, 2004; 4: 73-93.

4. Melone CP Jr, Beavers B, Isani A. The basal joint pain syndrome. Clin Orthop 1987; 220: 58-67.

5. Graham B, Regehr G, Naglie G, et al. Development and validation of diagnostic criteria for carpal tunnel syndrome. J Hand Surg Am, 2006; 31: 919.e1-919.e7.

6. Florack TM, Miller RJ, Pellegrini VD, et al. The prevalence of carpal tunnel syndrome in patients with basal joint arthritis of the thumb. J Hand Surg Am, 1992; 17: 624-30.

7. Le Viet D, Kerboull L, Lantieri $\mathrm{LA}$, et al. Stabilized resection arthroplasty by an anterior approach in trapeziometacarpal arthritis: results and surgical technique. J Hand Surg Am, 1996; 21: 194-201.

8. Stahl S, Shapira D. Trapeziometacarpal joint osteoartritis and carpal tunnel syndrome: anew surgical approach for concomitant treatment. J Hand Surg Br, 2003; 28: 246-50.
9. Cassidy C, Glennon PE, Stein $A B$, et al. Basal joint arthroplasty and carpal tunnel release through a single incision: an in vitro study. J Hand Surg Am, 2004; 29: 1085-8.

10. Weber RA, Sanders WE. Flexor carpi radialis approach for carpal tunnel release. J Hand Surg Am, 1997; 22: 120-6.

11. Eaton RG, Littler JW. Ligament reconstruction for the painful thumb carpometacarpal joint. J Bone Joint Surg Am, 1973; 55: 1655-66.

12. Burton RI, Pellegrini VD Jr. Surgical management of basal joint artritis of the thumb. Part I. Ligament reconstruction with tendon interposition arthroplasty. J Hand Surg Am, 1986; 11: 324-32.

13. Liang $\mathrm{MH}$, Fossel $\mathrm{AH}$, Larson MG. Comparisons of five status instruments for orthopaedic evaluation. Medical Care, 1990; 28: 632-42.

14. Torres-Coscoyuela M, González-del Pino J, Yánez-Calvo J, et al. Estudio dinamométrico de la mano y el pulgar. Rev Ortop Traumatol, 1999; 43: 3216.

15. Mathiowetz V, Kashman N, Volland $G$, et al. Grip and pinch strength: normative data for adults. Arch Phys Med Reh, 1985; 66: 69-72.

16. Rosales RS, Delgado EB, Diez de la Lastra-Bosch I. Evaluation of the Spanish version of the DASH and carpal tunnel syndrome health-related quality-of-live instruments: cross- cultural adaptation process and reliability. J Hand Surg Am, 2002; 27: 334-46.

17. De Smet, L. Responsiveness of the DASH score in surgical treated basal joint arthritis of the thumb: preliminary results. Clin Rheumatol, 2004; 23: 223-4.

18. Kazis L, Anderson JJ, Meenan RF. Effects sizes for interpreting changes in health status. Medical Care, 1989; 27 (Suppl 3): S18-S189.

19. Cohen J. Statistical power analysis for the behavioral sciences ( $2^{\mathrm{a}}$ ed.). Hillsdale, $\mathrm{NJ}$ : Lawrence Earlbaum Associates, 1988.

20. Dubert T, Kamoun L. Le tamisage des résultats, proposition d'un nouveau principe d'évaluations en chirurgie de la main. À propos d'une série de 72 libérations du canal carpien. Chir Main, 2005; 24: 305-9.

21. Nigro RO. Anatomy of the flexor retinaculum of the wrist and the flexor carpi radialis tunnel. Hand Clin, 2001; 17: 61-4.

22. Cooney WP III, Chao EY. Biomechanical analysis of static forces in the thumb during hand function. $J$ Bone Joint Surg Am, 1977; 59: 27-36.

23. Cobb TK, Dalley BK, Posteraro $\mathrm{RH}$, et al. Anatomy of the flexor retinaculum. J Hand Surg Am, 1993; 18: 91-9.

24. Lluch AL. Nerve compression. En: Kasdan ML, Amadio PC, Bowers WH (Eds). Technical tips for hand surgery. Philadel- 
phia: Hanley and Belfus, Inc., 1994: 169-70.

25. Sless Y, Sampson SP. Experience with transtrapezium approach for transverse carpal ligament release in patients with coexisted trapeziometacarpal joint osteoarthritis and carpal tunnel syndrome. Hand, 2007; 2: 151-4.
26. DaSilva MF, Moore DC, Weiss $\mathrm{AP}$, et al. Anatomy of the palmar cutaneous branch of the median nerve: clinical significance. J Hand Surg Am, 1996; 21: 639-43.

27. Martin CH, Seiler JG 3rd, Lesesne JS. The cutaneous innervation of the palm: an anato- mic study of the ulnar and median nerves. J Hand Surg Am, 1996; 21: 634-8.

28. Wheatley MJ, Hall JW, Faringer PD. Are the palmar cutaneous nerves safe during standard carpal tunnel release? Ann Plast Surg, 1996; 37: 251-3. 